\title{
Baire's Category Theorem and Some Spaces Generated from Real Normed Space ${ }^{1}$
}

\author{
Noboru Endou \\ Gifu National College of Technology \\ Gifu, Japan \\ Katsumasa Okamura \\ Shinshu University \\ Nagano, Japan
}

\author{
Yasunari Shidama \\ Shinshu University \\ Nagano, Japan
}

Summary. As application of complete metric space, we proved a Baire's category theorem. Then we defined some spaces generated from real normed space and discussed each of them. In the second section, we showed the equivalence of convergence and the continuity of a function. In other sections, we showed some topological properties of two spaces, which are topological space and linear topological space generated from real normed space.

MML identifier: NORMSP_2, version: 7.8.03 4.75.958

The papers [23], [7], [26], [4], [1], [21], [15], [27], [6], [5], [17], [19], [20], [24], [22], $[2],[25],[9],[10],[13],[16],[12],[11],[3],[18],[8]$, and [14] provide the notation and terminology for this paper.

\section{Baire's Category Theorem}

The following proposition is true

(1) Let $X$ be a non empty metric space and $Y$ be a sequence of subsets of $X$. Suppose $X$ is complete and $\bigcup \operatorname{rng} Y=$ the carrier of $X$ and for every element $n$ of $\mathbb{N}$ holds $Y(n)^{\mathrm{c}} \in$ the open set family of $X$. Then there exists an element $n_{0}$ of $\mathbb{N}$ and there exists a real number $r$ and there exists a point $x_{0}$ of $X$ such that $0<r$ and $\operatorname{Ball}\left(x_{0}, r\right) \subseteq Y\left(n_{0}\right)$.

\footnotetext{
${ }^{1}$ This work has been partially supported by the MEXT grant Grant-in-Aid for Young Scientists (B)16700156. 


\section{Metric Space Generated from Real Normed Space}

Let $X$ be a real normed space. The distance by norm of $X$ yields a function from : the carrier of $X$, the carrier of $X$ : into $\mathbb{R}$ and is defined as follows:

(Def. 1) For all points $x, y$ of $X$ holds (the distance by norm of $X)(x, y)=\|x-y\|$.

Let $X$ be a real normed space. The functor MetricSpaceNorm $X$ yields a non empty metric space and is defined by:

(Def. 2) MetricSpaceNorm $X=\langle$ the carrier of $X$, the distance by norm of $X\rangle$.

Next we state several propositions:

(2) Let $X$ be a real normed space, $z$ be an element of MetricSpaceNorm $X$, and $r$ be a real number. Then there exists a point $x$ of $X$ such that $x=z$ and $\operatorname{Ball}(z, r)=\{y ; y$ ranges over points of $X:\|x-y\|<r\}$.

(3) Let $X$ be a real normed space, $z$ be an element of MetricSpaceNorm $X$, and $r$ be a real number. Then there exists a point $x$ of $X$ such that $x=z$ and $\overline{\operatorname{Ball}}(z, r)=\{y ; y$ ranges over points of $X:\|x-y\| \leq r\}$.

(4) Let $X$ be a real normed space, $S$ be a sequence of $X, S_{1}$ be a sequence of MetricSpaceNorm $X, x$ be a point of $X$, and $x_{1}$ be a point of MetricSpaceNorm $X$. Suppose $S=S_{1}$ and $x=x_{1}$. Then $S_{1}$ is convergent to $x_{1}$ if and only if for every real number $r$ such that $0<r$ there exists an element $m$ of $\mathbb{N}$ such that for every element $n$ of $\mathbb{N}$ such that $m \leq n$ holds $\|S(n)-x\|<r$.

(5) Let $X$ be a real normed space, $S$ be a sequence of $X$, and $S_{1}$ be a sequence of MetricSpaceNorm $X$. If $S=S_{1}$, then $S_{1}$ is convergent iff $S$ is convergent.

(6) Let $X$ be a real normed space, $S$ be a sequence of $X$, and $S_{1}$ be a sequence of MetricSpaceNorm $X$. If $S=S_{1}$ and $S_{1}$ is convergent, then $\lim S_{1}=\lim S$.

\section{Topological Space Generated from Real Normed Space}

Let $X$ be a real normed space. The functor TopSpaceNorm $X$ yields a non empty topological space and is defined by:

(Def. 3) TopSpaceNorm $X=(\text { MetricSpaceNorm } X)_{\text {top }}$.

The following propositions are true:

(7) Let $X$ be a real normed space and $V$ be a subset of TopSpaceNorm $X$. Then $V$ is open if and only if for every point $x$ of $X$ such that $x \in V$ there exists a real number $r$ such that $r>0$ and $\{y ; y$ ranges over points of $X$ : $\|x-y\|<r\} \subseteq V$. 
(8) Let $X$ be a real normed space, $x$ be a point of $X$, and $r$ be a real number. Then $\{y ; y$ ranges over points of $X:\|x-y\|<r\}$ is an open subset of TopSpaceNorm $X$.

(9) Let $X$ be a real normed space, $x$ be a point of $X$, and $r$ be a real number. Then $\{y ; y$ ranges over points of $X:\|x-y\| \leq r\}$ is a closed subset of TopSpaceNorm $X$.

(10) For every Hausdorff non empty topological space $X$ such that $X$ is locally-compact holds $X$ is Baire.

(11) For every real normed space $X$ holds TopSpaceNorm $X$ is sequential.

Let $X$ be a real normed space. Observe that TopSpaceNorm $X$ is sequential. One can prove the following propositions:

(12) Let $X$ be a real normed space, $S$ be a sequence of $X, S_{1}$ be a sequence of TopSpaceNorm $X, x$ be a point of $X$, and $x_{1}$ be a point of TopSpaceNorm $X$. Suppose $S=S_{1}$ and $x=x_{1}$. Then $S_{1}$ is convergent to $x_{1}$ if and only if for every real number $r$ such that $0<r$ there exists an element $m$ of $\mathbb{N}$ such that for every element $n$ of $\mathbb{N}$ such that $m \leq n$ holds $\|S(n)-x\|<r$.

(13) Let $X$ be a real normed space, $S$ be a sequence of $X$, and $S_{1}$ be a sequence of TopSpaceNorm $X$. If $S=S_{1}$, then $S_{1}$ is convergent iff $S$ is convergent.

(14) Let $X$ be a real normed space, $S$ be a sequence of $X$, and $S_{1}$ be a sequence of TopSpaceNorm $X$. If $S=S_{1}$ and $S_{1}$ is convergent, then $\operatorname{Lim} S_{1}=$ $\{\lim S\}$ and $\lim S_{1}=\lim S$.

(15) Let $X$ be a real normed space, $V$ be a subset of $X$, and $V_{1}$ be a subset of TopSpaceNorm $X$. If $V=V_{1}$, then $V$ is closed iff $V_{1}$ is closed.

(16) Let $X$ be a real normed space, $V$ be a subset of $X$, and $V_{1}$ be a subset of TopSpaceNorm $X$. If $V=V_{1}$, then $V$ is open iff $V_{1}$ is open.

(17) Let $X$ be a real normed space, $U$ be a subset of $X, U_{1}$ be a subset of TopSpaceNorm $X, x$ be a point of $X$, and $x_{1}$ be a point of TopSpaceNorm $X$. Suppose $U=U_{1}$ and $x=x_{1}$. Then $U$ is a neighbourhood of $x$ if and only if $U_{1}$ is a neighbourhood of $x_{1}$.

(18) Let $X, Y$ be real normed spaces, $f$ be a partial function from $X$ to $Y, f_{1}$ be a function from TopSpaceNorm $X$ into TopSpaceNorm $Y, x$ be a point of $X$, and $x_{1}$ be a point of TopSpaceNorm $X$. Suppose $f=f_{1}$ and $x=x_{1}$. Then $f$ is continuous in $x$ if and only if $f_{1}$ is continuous at $x_{1}$.

(19) Let $X, Y$ be real normed spaces, $f$ be a partial function from $X$ to $Y$, and $f_{1}$ be a function from TopSpaceNorm $X$ into TopSpaceNorm $Y$. Suppose $f=f_{1}$. Then $f$ is continuous on the carrier of $X$ if and only if $f_{1}$ is continuous. 


\section{Linear Topological Space Generated from Real Normed Space}

Let $X$ be a real normed space. The functor LinearTopSpaceNorm $X$ yields a strict non empty real linear topological structure and is defined by the conditions (Def. 4).

(Def. 4)(i) The carrier of LinearTopSpaceNorm $X=$ the carrier of $X$,

(ii) the zero of LinearTopSpaceNorm $X=$ the zero of $X$,

(iii) the addition of LinearTopSpaceNorm $X=$ the addition of $X$,

(iv) the external multiplication of LinearTopSpaceNorm $X=$ the external multiplication of $X$, and

(v) the topology of LinearTopSpaceNorm $X=$ the topology of TopSpaceNorm $X$.

Let $X$ be a real normed space. Note that LinearTopSpaceNorm $X$ is addcontinuous, mult-continuous, topological space-like, Abelian, add-associative, right zeroed, right complementable, and real linear space-like.

We now state several propositions:

(20) Let $X$ be a real normed space, $V$ be a subset of TopSpaceNorm $X$, and $V_{1}$ be a subset of LinearTopSpaceNorm $X$. If $V=V_{1}$, then $V$ is open iff $V_{1}$ is open.

(21) Let $X$ be a real normed space, $V$ be a subset of TopSpaceNorm $X$, and $V_{1}$ be a subset of LinearTopSpaceNorm $X$. If $V=V_{1}$, then $V$ is closed iff $V_{1}$ is closed.

(22) Let $X$ be a real normed space and $V$ be a subset of LinearTopSpaceNorm $X$. Then $V$ is open if and only if for every point $x$ of $X$ such that $x \in V$ there exists a real number $r$ such that $r>0$ and $\{y ; y$ ranges over points of $X:\|x-y\|<r\} \subseteq V$.

(23) Let $X$ be a real normed space, $x$ be a point of $X, r$ be a real number, and $V$ be a subset of LinearTopSpaceNorm $X$. If $V=\{y ; y$ ranges over points of $X:\|x-y\|<r\}$, then $V$ is open.

(24) Let $X$ be a real normed space, $x$ be a point of $X, r$ be a real number, and $V$ be a subset of TopSpaceNorm $X$. If $V=\{y ; y$ ranges over points of $X:\|x-y\| \leq r\}$, then $V$ is closed.

Let $X$ be a real normed space. Observe that LinearTopSpaceNorm $X$ is $T_{2}$ and LinearTopSpaceNorm $X$ is sober.

One can prove the following proposition

(25) Let $X$ be a real normed space, $S$ be a family of subsets of TopSpaceNorm $X, S_{1}$ be a family of subsets of LinearTopSpaceNorm $X, x$ be a point of TopSpaceNorm $X$, and $x_{1}$ be a point of LinearTopSpaceNorm $X$. Suppose $S=S_{1}$ and $x=x_{1}$. Then $S_{1}$ is a basis of $x_{1}$ if and only if $S$ is a basis of $x$. 
Let $X$ be a real normed space. One can verify the following observations:

* LinearTopSpaceNorm $X$ is first-countable,

* LinearTopSpaceNorm $X$ is Frechet, and

* LinearTopSpaceNorm $X$ is sequential.

Next we state a number of propositions:

(26) Let $X$ be a real normed space, $S$ be a sequence of TopSpaceNorm $X$, $S_{1}$ be a sequence of LinearTopSpaceNorm $X, x$ be a point of TopSpaceNorm $X$, and $x_{1}$ be a point of LinearTopSpaceNorm $X$. Suppose $S=S_{1}$ and $x=x_{1}$. Then $S_{1}$ is convergent to $x_{1}$ if and only if $S$ is convergent to $x$.

(27) Let $X$ be a real normed space, $S$ be a sequence of TopSpaceNorm $X$, and $S_{1}$ be a sequence of LinearTopSpaceNorm $X$. If $S=S_{1}$, then $S_{1}$ is convergent iff $S$ is convergent.

(28) Let $X$ be a real normed space, $S$ be a sequence of TopSpaceNorm $X$, and $S_{1}$ be a sequence of LinearTopSpaceNorm $X$. If $S=S_{1}$ and $S_{1}$ is convergent, then $\operatorname{Lim} S=\operatorname{Lim} S_{1}$ and $\lim S=\lim S_{1}$.

(29) Let $X$ be a real normed space, $S$ be a sequence of $X, S_{1}$ be a sequence of LinearTopSpaceNorm $X, x$ be a point of $X$, and $x_{1}$ be a point of LinearTopSpaceNorm $X$. Suppose $S=S_{1}$ and $x=x_{1}$. Then $S_{1}$ is convergent to $x_{1}$ if and only if for every real number $r$ such that $0<r$ there exists an element $m$ of $\mathbb{N}$ such that for every element $n$ of $\mathbb{N}$ such that $m \leq n$ holds $\|S(n)-x\|<r$.

(30) Let $X$ be a real normed space, $S$ be a sequence of $X$, and $S_{1}$ be a sequence of LinearTopSpaceNorm $X$. If $S=S_{1}$, then $S_{1}$ is convergent iff $S$ is convergent.

(31) Let $X$ be a real normed space, $S$ be a sequence of $X$, and $S_{1}$ be a sequence of LinearTopSpaceNorm $X$. If $S=S_{1}$ and $S_{1}$ is convergent, then $\operatorname{Lim} S_{1}=\{\lim S\}$ and $\lim S_{1}=\lim S$.

(32) Let $X$ be a real normed space, $V$ be a subset of $X$, and $V_{1}$ be a subset of LinearTopSpaceNorm $X$. If $V=V_{1}$, then $V$ is closed iff $V_{1}$ is closed.

(33) Let $X$ be a real normed space, $V$ be a subset of $X$, and $V_{1}$ be a subset of LinearTopSpaceNorm $X$. If $V=V_{1}$, then $V$ is open iff $V_{1}$ is open.

(34) Let $X$ be a real normed space, $U$ be a subset of TopSpaceNorm $X, U_{1}$ be a subset of LinearTopSpaceNorm $X, x$ be a point of TopSpaceNorm $X$, and $x_{1}$ be a point of LinearTopSpaceNorm $X$. Suppose $U=U_{1}$ and $x=x_{1}$. Then $U$ is a neighbourhood of $x$ if and only if $U_{1}$ is a neighbourhood of $x_{1}$.

(35) Let $X, Y$ be real normed spaces, $f$ be a function from TopSpaceNorm $X$ into TopSpaceNorm $Y, f_{1}$ be a function from LinearTopSpaceNorm $X$ into LinearTopSpaceNorm $Y, x$ be a point of TopSpaceNorm $X$, and $x_{1}$ be a 
point of LinearTopSpaceNorm $X$. Suppose $f=f_{1}$ and $x=x_{1}$. Then $f$ is continuous at $x$ if and only if $f_{1}$ is continuous at $x_{1}$.

(36) Let $X, Y$ be real normed spaces, $f$ be a function from TopSpaceNorm $X$ into TopSpaceNorm $Y$, and $f_{1}$ be a function from LinearTopSpaceNorm $X$ into LinearTopSpaceNorm $Y$. If $f=f_{1}$, then $f$ is continuous iff $f_{1}$ is continuous.

\section{REFERENCES}

[1] Grzegorz Bancerek. The ordinal numbers. Formalized Mathematics, 1(1):91-96, 1990.

[2] Grzegorz Bancerek. The "way-below" relation. Formalized Mathematics, 6(1):169-176, 1997.

[3] Leszek Borys. Paracompact and metrizable spaces. Formalized Mathematics, 2(4):481485, 1991.

[4] Czesław Byliński. Functions and their basic properties. Formalized Mathematics, 1(1):5565, 1990.

[5] Czesław Byliński. Functions from a set to a set. Formalized Mathematics, 1(1):153-164, 1990.

6] Czesław Byliński. Partial functions. Formalized Mathematics, 1(2):357-367, 1990.

[7] Czesław Byliński. Some basic properties of sets. Formalized Mathematics, 1(1):47-53, 1990.

[8] Czesław Byliński. Introduction to real linear topological spaces. Formalized Mathematics, 13(1):99-107, 2005.

[9] Agata Darmochwał. Compact spaces. Formalized Mathematics, 1(2):383-386, 1990.

[10] Alicia de la Cruz. Totally bounded metric spaces. Formalized Mathematics, 2(4):559-562, 1991.

[11] Stanisława Kanas and Adam Lecko. Sequences in metric spaces. Formalized Mathematics, 2(5):657-661, 1991.

[12] Stanisława Kanas, Adam Lecko, and Mariusz Startek. Metric spaces. Formalized Mathematics, 1(3):607-610, 1990.

[13] Zbigniew Karno. Continuity of mappings over the union of subspaces. Formalized Mathematics, 3(1):1-16, 1992.

[14] Takaya Nishiyama, Keiji Ohkubo, and Yasunari Shidama. The continuous functions on normed linear spaces. Formalized Mathematics, 12(3):269-275, 2004.

[15] Beata Padlewska. Families of sets. Formalized Mathematics, 1(1):147-152, 1990.

[16] Beata Padlewska. Locally connected spaces. Formalized Mathematics, 2(1):93-96, 1991.

[17] Beata Padlewska and Agata Darmochwal. Topological spaces and continuous functions. Formalized Mathematics, 1(1):223-230, 1990.

[18] Jan Popiołek. Real normed space. Formalized Mathematics, 2(1):111-115, 1991.

[19] Bartłomiej Skorulski. First-countable, sequential, and Frechet spaces. Formalized Mathematics, 7(1):81-86, 1998.

[20] Bartłomiej Skorulski. The sequential closure operator in sequential and Frechet spaces. Formalized Mathematics, 8(1):47-54, 1999.

[21] Andrzej Trybulec. Subsets of complex numbers. To appear in Formalized Mathematics.

[22] Andrzej Trybulec. Domains and their Cartesian products. Formalized Mathematics, 1(1):115-122, 1990.

[23] Andrzej Trybulec. Tarski Grothendieck set theory. Formalized Mathematics, 1(1):9-11, 1990.

[24] Andrzej Trybulec. Baire spaces, Sober spaces. Formalized Mathematics, 6(2):289-294, 1997.

[25] Wojciech A. Trybulec. Vectors in real linear space. Formalized Mathematics, 1(2):291$296,1990$.

[26] Zinaida Trybulec. Properties of subsets. Formalized Mathematics, 1(1):67-71, 1990. 
[27] Edmund Woronowicz. Relations defined on sets. Formalized Mathematics, 1(1):181-186, 1990.

Received November 21, 2006 